\title{
ANALYSIS OF MEMBRANE HETEROGENEITY FOR THE ADSORPTIVE MEMBRANE SEPARATOR
}

\author{
MOTONOBU GOTO AND TSUTOMU HIROSE \\ Department of Applied Chemistry, Kumamoto University, \\ Kumamoto 860
}

Key Words: Adsorption, Membrane, Heterogeneity, Moment, Porosity, Axial Dispersion

\begin{abstract}
Adsorptive membranes have been recently developed for affinity or chelating separation to reduce diffusion resistance, which is a major factor in broadening of the breakthrough or elution curve. Since the axial distance along the fluid flow is relatively short, the heterogeneity in the membrane is expected to influence the performance of the adsorption. The heterogeneities in membrane structure such as porosity, thickness and pore size are evaluated theoretically by moment analysis. The first, second and third moments are shown as a function of heterogeneity such as porosity distribution. The parameters involved in the system are adsorption equilibrium constant, Peclet number, and adsorption rate constant. The influences of these parameters as well as heterogeneity are evaluated. The heterogeneity produced unfavorable peak broadening and skewness.
\end{abstract}

\section{Introduction}

With the development of support materials for chromatography, chromatographic separation has become widely used in a variety of fields for preparative purposes as well as an analytical tool. Although the adsorption is usually carried out in a packed-bed column, various adsorber geometries have been studied to improve separator performance.

The performance of the adsorber is related to mass transfer and adsorption kinetics. Mass transfer resistance within the adsorbent in a column can be reduced by using smaller particles, which eventually requires a shorter and wider bed to maintain low pressure drops and high capacity. Another way to reduce the diffusion distance is to utilize the convection effect in the adsorbent pore by using an adsorbent with large pores ${ }^{10}$. Consequently, the ideal geometry of the bed could be realized by a membrane-type adsorbent. A shorter flow path would minimize operation pressures and maximize throughput. A larger cross-sectional area would maximize loading and adsorption capacity. Mass transfer limitation could be avoided by convection of the fluid in the pores of the membrane.

When the adsorption isotherm is highly favorable, solutes are efficiently adsorbed even by a thin membrane. Separation by adsorbents with highly favorable adsorption isotherms is often carried out by a series of operating processes consisting of an adsorption step followed by a desorption step where the fluid properties are changed for elution. Affinity adsorption and chelating adsorption are examples of this type.

A chelating membrane adsorbent in hollow-fiber geometry has been developed ${ }^{7)}$. The shape of the breakthrough curves was not dependent on the liquid resi-

\footnotetext{
* Received March 15, 1993. Correspondence concerning this article should be addressed to M. Goto.
}

dence time and the overall adsorption rate was not governed by the diffusion resistance.

An affinity adsorbent in membrane geometry termed affinity membrane has also been developed ${ }^{6}$. The capacity can be increased by a column consisting of a stack of membranes ${ }^{5)}$ or hollow-fiber configuration ${ }^{4)}$. Since the affinity between biological molecules is strong, the adsorption isotherm is highly favorable. In that system, biological molecules of interest can be efficiently captured by the adsorbent in spite of the short residence time within the adsorbent bed.

Suen and Etzel ${ }^{11)}$ calculated breakthrough curves of affinity membrane by a mathematical model. The effects of axial diffusion, flow velocity and association kinetics were discussed. The performance of the membrane separator might be sensitive to the heterogeneity of the membrane. Suen and Etzel ${ }^{11)}$ showed that variation in porosity or membrane thickness caused considerable broadening of the breakthrough curve in the case of negligible axial dispersion $(P e=\infty)$. There are many factors where heterogeneity exists; e. g., porosity, thickness, ligand density, association kinetics and pore size. $\mathrm{McCoy}^{9)}$ developed a mathematical analysis for chromatography of a heterogeneous mixture where the adsorption binding constants had a distribution. Moment analysis was used to reveal the effect of heterogeneity on the adsorption equilibrium constant.

The present objective is to study the effect of heterogeneity in membrane structure on the performance of the adsorptive membrane separator. The moment analysis developed by $\mathrm{McCoy}^{9)}$ is extended to the heterogeneity in porosity, thickness, or pore size of the membrane. McCoy9) analyzed the system where the solute has a distribution of binding constant, while we deal with the system where the adsorber has a distribu- 
tion of structural properties. Distinctive features of the membrane separator are the absence of diffusion resistance and short axial distance, which may result in enlarged influence of the heterogeneity. The analyses give us insight into these effects, which may permit us to design a high-performance membrane separator.

\section{Theory}

\subsection{Model equations}

The main rate-controlling step, which causes band broadening in conventional chromatography, may arise from convective flow, intraparticle diffusion, external mass transfer, axial dispersion or association rate. Since there exists convective flow within pores of the membrane, no diffusion limitation, which is a major rate-controlling step for the conventional packed-bed adsorber, exists there.

A mass balance over a section of the membrane for a single-solute system gives the following continuity equation $^{11)}$ :

$$
\varepsilon \frac{\partial c}{\partial t}+\varepsilon v \frac{\partial c}{\partial z}=\varepsilon D_{a} \frac{\partial^{2} c}{\partial z^{2}}-(1-\varepsilon) \frac{\partial c_{s}}{\partial t}
$$

where the solute concentration adsorbed is based on the solid phase volume. The association rate between solute molecule and adsorption site (ligand) on the adsorbent can often be expressed by the reversible second-order rate expression

$$
\frac{\partial c_{s}}{\partial t}=k_{a}^{\prime} c\left(c_{l}-c_{s}\right)-k_{d} c_{s}
$$

In the equilibrium case, this rate equation reduces to a Langmuir isotherm.

$$
c_{s}=\frac{\left(k_{a}^{\prime} / k_{d}\right) c_{l} c}{1+\left(k_{a} / k_{d}\right) c}
$$

When the concentration of solute is low enough as compared with ligand concentration, or association is not so specific, the association rate equation can be simplified to linear form.

$$
\frac{\partial c_{s}}{\partial t}=k_{a}\left(c-\frac{c_{s}}{K}\right)
$$

where $k_{a}=c_{l} k_{a}$ '. When the linear rate expression, which corresponds to a linear adsorption isotherm, is used, mathematical analyses can be significantly simplified. Although the linear case is rare for affinity separation in the adsorption step ${ }^{1)}$, the desorption (elution) step is usually operated under less favorable or linear condition. Band broadening in the adsorption step influences the adsorption efficiency, while that in the desorption step influences the resolution or separation among adsorbed components. Consequently, the following discussion is restricted to the linear case. The conclusion derived for the linear case may be applicable to the nonlinear case.

The initial and boundary conditions for an impulse function are

$$
\begin{gathered}
c(t=0)=0 \\
c_{s}(t=0)=0 \\
c(z=0)=M \delta(t) \\
c(z=\infty)=\text { finite }
\end{gathered}
$$

These equations, Eqs.(1), (4), and (5)-(8) can be solved by means of a Laplace transform. The solution for $c$ in the Laplace domain is given by

$$
\hat{c}=M \exp \left\{\frac{1}{2}\left[\frac{v}{D_{a}}-\sqrt{\left(\frac{v}{D_{a}}\right)^{2}+\frac{4}{D_{a}}\left(s+\frac{1-\varepsilon}{\varepsilon} \frac{k_{a} s}{s+k_{a} / K}\right)}\right] z\right\}
$$

\subsection{Heterogeneity in porosity}

For a packed bed of adsorbent beads, the most influential factor for band broadening is intraparticle diffusion, although the association rate is often important for band broadening in the case of affinity adsorption. On the other hand, since no diffusion resistance in pores exists in the membrane adsorber, the chromatographic elution curve or breakthrough (frontal) curve is expected to be sharp. Since the filtration area or cross-sectional area for liquid flow is relatively large compared with the length in the flow direction, the effect of heterogeneity of membrane might be increased. Heterogeneities of the membrane are expected to exist in porosity, pore size, ligand density, association kinetics, thickness, etc.

Here we consider heterogeneity in porosity, $\varepsilon$, of the membrane. The governing differential equation for the portion having specific porosity, $\varepsilon_{\mathrm{i}}$, can be given as:

$$
\varepsilon_{i} \frac{\partial c_{i}}{\partial t}+\varepsilon_{i} v_{i} \frac{\partial c_{i}}{\partial z}=\varepsilon_{i} D_{a} \frac{\partial^{2} c_{i}}{\partial z^{2}}-\left(1-\varepsilon_{i}\right) \frac{\partial c_{s, i}}{\partial t}
$$

Since heterogeneity in porosity influences the flow rate in the pore, interstitial fluid velocity becomes a function of $\varepsilon_{i}$. When the pressure drop of membranes is expressed by the Blake-Kozeny equation ${ }^{3)}$ given by

$$
\Delta P=150 \nu L \mu(1-\varepsilon)^{2} /\left(D_{P}^{2} \varepsilon^{2}\right)
$$

the interstitial velocity can be expressed by

$$
v_{i}=\left(\frac{\varepsilon_{\mathrm{i}}}{1-\varepsilon_{i}}\right)^{2}\left(\frac{1-\varepsilon_{0}}{\varepsilon_{0}}\right)^{2} v_{0}
$$

where $\mathrm{v}_{0}$ is the value at porosity $\varepsilon_{0}$.

\subsection{Moment equations}

The moment expressions are obtained from the 
Laplace transform solution by the relation

$$
m_{n}=(-1)^{n} \lim _{s \rightarrow 0} \frac{d^{n} \hat{c}}{d s^{n}}
$$

The zero, first, second and third moments for the portion $i$ whose porosity is $\varepsilon_{i}$ are obtained as follows.

$$
\begin{gathered}
m_{0 i}(z)=m_{0 i}(z=0)=M_{i} \\
m_{1 i}(z)=m_{0 i} \frac{\theta}{A_{0}} \frac{A_{i}}{B_{i}} \\
m_{2 i}(z)=m_{0 i}\left(\frac{\theta}{A_{0}}\right)^{2}\left(\frac{2 K^{2}}{\phi} \frac{E_{i}}{B_{i}}+\frac{2}{P e} \frac{A_{i}^{2}}{B_{i}^{3}}+\frac{A_{i}^{2}}{B_{i}{ }^{2}}\right) \\
m_{3 i}(z)=m_{0 i}\left(\frac{\theta}{A_{0}}\right)^{3}\left(\frac{6 K^{3}}{\phi^{2}} \frac{E_{i}}{B_{i}}+\frac{12 K^{2}}{P e \phi} \frac{E_{i} A_{i}}{B_{i}^{3}}+\frac{6 K^{2}}{\phi} \frac{E_{i} A_{i}}{B_{i}{ }^{2}}\right. \\
\left.+\frac{12}{P e^{2}} \frac{A_{i}^{3}}{B_{i}^{5}}+\frac{6}{P e} \frac{A_{i}^{3}}{B_{i}^{4}}+\frac{A_{i}^{3}}{B_{i}^{3}}\right)
\end{gathered}
$$

where $E_{i}=\left(1-\varepsilon_{i}\right) / \varepsilon_{i}, E_{0}=\left(1-\varepsilon_{0}\right) / \varepsilon_{0}, A_{\mathrm{i}}=1+E_{\mathrm{i}} K$, $A_{0}=1+E_{0} K, B_{\mathrm{i}}=\left(E_{0} / E_{i}\right)^{2}, P e=z v_{0} / D_{a}, \theta=A_{0} z / v_{0}$, and $\phi=k_{a} z / v_{0}$. These symbolic computations as well as the following numeric calculations were done with a computer algebra system ${ }^{2)}$ (Mathematica $^{\mathrm{TM}}$, Wolfram Research Inc.)

When the heterogeneity of the membrane gives continuous porosity variation, the total concentration is given by the integral over the variable $\xi$, which can be regarded as the positional coordinate of the membrane.

$$
c(t, z)=\int c(\xi, t, z) v(\xi) d \xi / \int v(\xi) d \xi
$$

Then the temporal moments can be expressed as

$$
m_{n}(z)=\int m_{n}(\xi, z) v(\xi) d \xi / \int v(\xi) d \xi
$$

where

$$
m_{n}(\xi, z)=\int t^{n} c(\xi, t, z) d t
$$

The zero moment of the inlet pulse is obtained from the boundary condition at $z=0$.

$$
\begin{aligned}
m_{0}(z) & =m_{0}(0)=\int m_{0}(\xi, 0) v(\xi) d \xi / \int v(\xi) d \xi \\
& =\int M(\xi) v(\xi) d \xi / \int v(\xi) d \xi
\end{aligned}
$$

The first absolute moment, which represents the retention time, is

$$
\mu_{1}^{\prime}(z)=\frac{m_{1}(z)}{m_{0}(z)}=\frac{\theta}{A_{0}}\left\langle\frac{A}{B}\right\rangle
$$

where $\langle A / B\rangle$ is the average value of $A(\xi) / B(\xi)$. The average value of a function $F(\xi)$ is given by

$$
\langle F\rangle=\int F(\xi) M(\xi) v(\xi) d \xi / \int M(\xi) v(\xi) d \xi
$$

In the above case, $F(\xi)=A(\xi) / B(\xi)$.

The $n$-th central moment is defined as

$$
\mu_{n}=\sum_{i=0}^{n}\left(\begin{array}{c}
n \\
i
\end{array}\right) \frac{m_{i}}{m_{0}}\left(-\frac{m_{1}}{m_{0}}\right)^{n-i}
$$

The second central moment, which represents the variance, is then given by

$$
\begin{aligned}
\mu_{2}(z)=\left(\frac{\theta}{A_{0}}\right)^{2} \frac{2}{P e}\{\langle & \left\langle\frac{A^{2}}{B^{3}}\right\rangle+\frac{P e K^{2}}{\phi}\left\langle\frac{E}{B}\right\rangle \\
& \left.+\frac{P e}{2}\left[\left\langle\frac{A^{2}}{B^{2}}\right\rangle-\left\langle\frac{A}{B}\right\rangle^{2}\right]\right\}
\end{aligned}
$$

The term in square brackets [ ] indicates a contribution to band broadening due solely to heterogeneity, because the term $\left.\left[<A^{2} / B^{2}>-<A / B\right\rangle^{2}\right]$ disappears without heterogeneity.

The third central moment, which represents skewness, is given by

$$
\begin{aligned}
& \mu_{3}(z)=\left(\frac{\theta}{A_{0}}\right)^{3} \frac{12}{P e^{2}}\left\{\left\langle\frac{A^{3}}{B^{5}}\right\rangle+\frac{P e^{2} K^{2}}{2 \phi}\right. \\
&\left(\frac{K}{\phi}\left\langle\frac{E}{B}\right\rangle+\frac{2}{P e}\left\langle\frac{E A}{B^{3}}\right\rangle+\left[\left\langle\frac{E A}{B^{2}}\right\rangle-\left\langle\frac{E}{A}\right\rangle\left\langle\frac{A}{B}\right\rangle\right]\right) \\
&+\frac{P e}{2}\left[\left\langle\frac{A^{3}}{B^{4}}\right\rangle-\left\langle\frac{A^{2}}{B^{3}}\right\rangle\left\langle\frac{A}{B}\right\rangle\right]+\frac{P e^{2}}{12} \\
& {\left.\left[\left\langle\frac{A^{3}}{B^{3}}\right\rangle-3\left\langle\frac{A^{2}}{B^{2}}\right\rangle\left\langle\frac{A}{B}\right\rangle+2\left\langle\frac{A}{B}\right\rangle^{3}\right]\right\} }
\end{aligned}
$$

The terms in square brackets [ ] are the contribution of the heterogeneity.

When the adsorption and desorption rate are rapid, local equilibrium can be assumed, that is, $k_{a}$ is infinitely large and then $\phi=\infty$. In that case the second and third moment expressions are reduced to:

$$
\mu_{2}(z)=\left(\frac{\theta}{A_{0}}\right)^{2} \frac{2}{P e}\left\{\left\langle\frac{A^{2}}{B^{3}}\right\rangle+\frac{P e}{2}\left[\left\langle\frac{A^{2}}{B^{2}}\right\rangle-\left\langle\frac{A}{B}\right\rangle^{2}\right]\right\}
$$



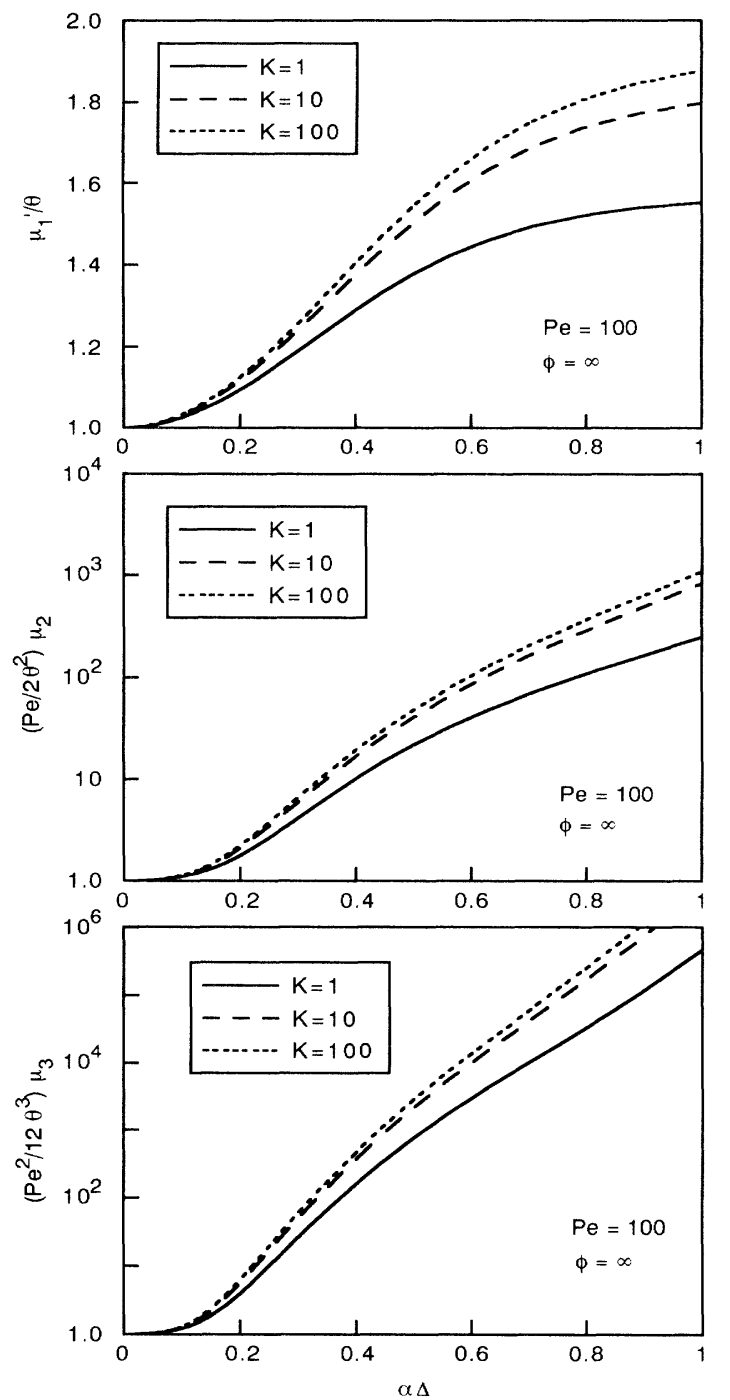

Fig. 1 First, second and third moments for Gaussian distribution of porosity at different values of $K$

$$
\begin{array}{r}
\mu_{3}(z)=\left(\frac{\theta}{A_{0}}\right)^{3} \frac{12}{P e^{2}}\left\{\left\langle\frac{A^{3}}{B^{5}}\right\rangle+\frac{P e}{2}\left[\left\langle\frac{A^{3}}{B^{4}}\right\rangle-\left\langle\frac{A^{2}}{B^{3}}\right\rangle\left\langle\frac{A}{B}\right\rangle\right]\right. \\
\left.+\frac{P e^{2}}{12}\left[\left\langle\frac{A^{3}}{B^{3}}\right\rangle-3\left\langle\frac{A^{2}}{B^{2}}\right\rangle\left\langle\frac{A}{B}\right\rangle+2\left\langle\frac{A}{B}\right\rangle^{3}\right]\right\}
\end{array}
$$

For the local equilibrium case, the solution in the time domain $^{8)}$ is given by

$$
\frac{c \theta}{M}=\frac{B_{i}}{2} \sqrt{\frac{P e A_{0} \theta}{\pi A t}} \exp \left[-\frac{P e A_{0} B_{i}^{2} \theta}{4 A t}\left(\frac{t}{\theta}-\frac{A_{i}}{A B}\right)^{2}\right]
$$

The average value $\left\langle c \theta / M_{0}\right\rangle$ as a function of $t / \theta$ can be calculated in the same manner by Eq. (23).

\subsection{Gaussian distribution}

We have two distribution functions in the above equations, $\varepsilon(\xi)$ and $M(\xi)$. For a chromatographic impulse injected into the upstream side of the membrane, the total amount of solute, $M(\xi)$, entereing at the portion


Fig. 2 First, second and third moments at different values of $\mathrm{Pe}$

of $\xi$ must vary with porosity. If it is assumed that $M(\xi)$ is proportional to the porosity and interstitial velocity, $M(\xi)$ is given as a function of $\varepsilon(\xi)$ by

$$
M(\xi)=M_{0} \frac{\varepsilon(\xi) \nu(\xi)}{\varepsilon_{0} v_{0}}=M_{0} \frac{\varepsilon(\xi)^{3}}{(1-\varepsilon(\xi))^{2}} \frac{\left(1-\varepsilon_{0}\right)^{2}}{\varepsilon_{0}^{3}}
$$

Consider the Gaussian distribution of porosity:

$$
\varepsilon(\xi)=\varepsilon_{0} \exp \left(-\alpha^{2} \xi^{2}\right) \quad \text { for }|\xi|<\Delta
$$

The average values $<>$ appearing in Eqs. (22) and (25)(28) can be calculated by integration in terms of $\xi$ over the range from 0 to to $\alpha \Delta$. The moments, $\mu_{1}{ }^{\prime}, \mu_{2}$ and $\mu_{3}$, can be evaluated for various values of $\alpha \Delta$ by substituting average values $<>$. The degree of heterogeneity depends on the integration range $\alpha \Delta$. When $\alpha \Delta$ is large, the heterogeneity is high. On the other hand, when $\alpha \Delta$ approaches zero, the membrane can be considered to be 


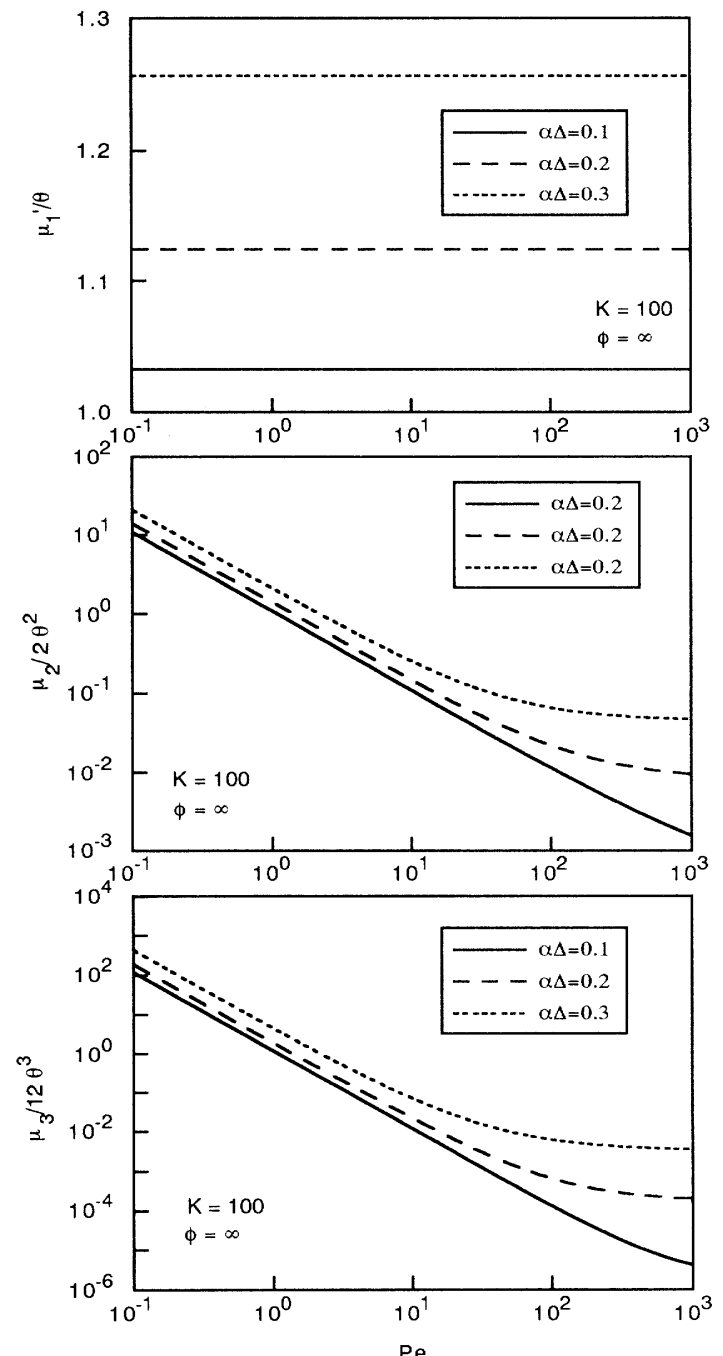

Fig. 3 Plots of $\mu_{1}{ }^{\prime} / \theta, \mu_{2} / 2 \theta^{2}$ and $\mu_{3} / 12 \theta^{3}$ as a function of $\mathrm{Pe}$

homogeneous.

The Gaussian distribution of porosity as a function of $\xi$ given by Eq. (31) is not identical to the Gaussian frequency distribution of porosity. The frequency distribution is given by $d \xi / d \varepsilon$ from Eq. (31).

\section{Results and Discussion}

\subsection{Effect of porosity heterogeneity}

The moments were calculated in the equilibrium case $(\phi=\infty)$ by using Eqs. (22), (27) and (28). The parameters involved in the calculation were $\varepsilon_{0}, K$ and $P e$. The values adopted for these parameters are $\varepsilon_{0}=0.7, K=$ $0.1-1000$, and $P e=0.1-1000$. The evaluated moments are shown in Fig. 1 as $\mu_{1} / \theta,\left(\mathrm{Pe} / 2 \theta^{2}\right) \mu_{2}$, and $\left(P e^{2} /\right.$ $\left.12 \theta^{3}\right) \mu_{3}$ versus $\alpha \Delta$ for $P e=100$ where the influence of axial dispersion is insignificant. Without heterogeneity these values are unity for the equilibrium case. The variation of porosity defined by $1-\varepsilon(\Delta) / \varepsilon_{0}$ for $0.01,0.1,1$, 5 , and $10 \%$ corresponds to $\alpha \Delta=0.01,0.032,0.10,0.23$, and 0.32 , respectively. Figure 1 indicates that a heterogeneity of $5 \%$ generates about $1.2,3.0$, and 13 times
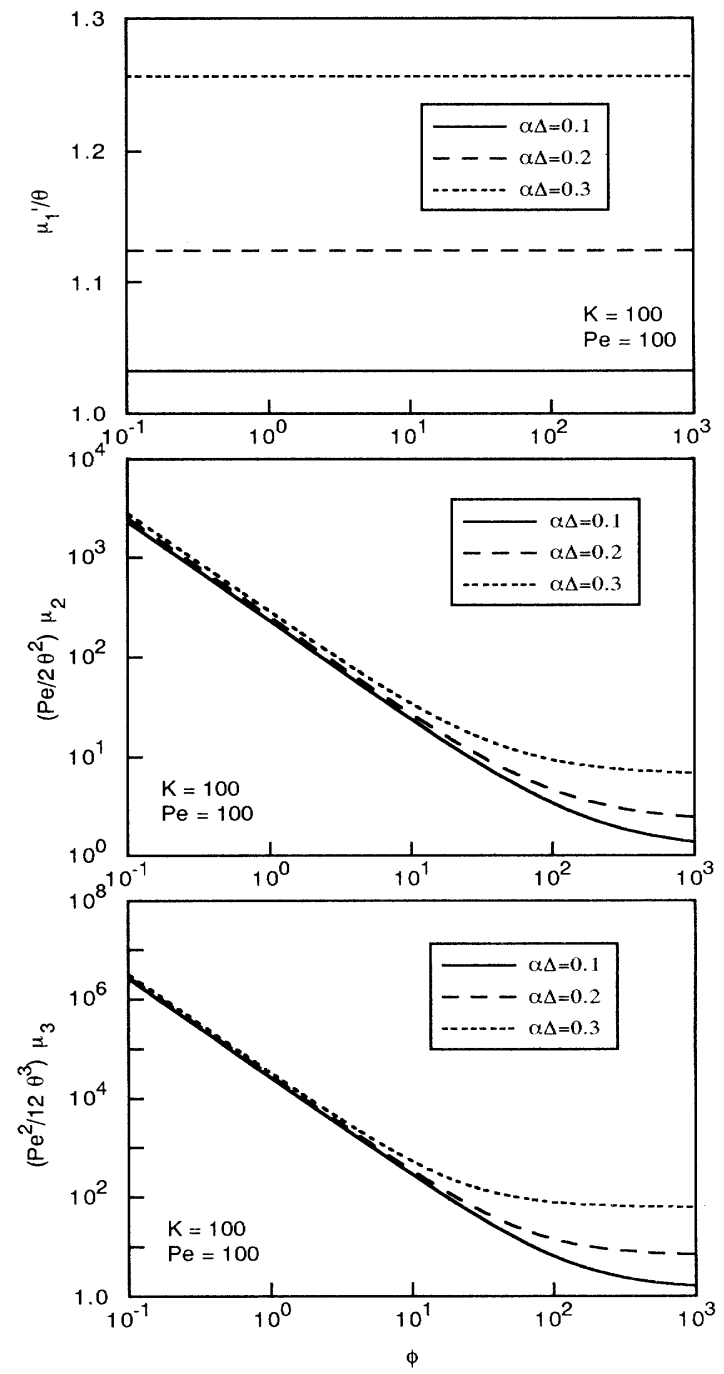

Fig. 4 Effect of adsorption rate on the moments at different values of $\alpha \Delta$

increase in first, second, and third moments respectively when $P e=100$ and $K=100$, and they are about 1.3, 8.4, and 96 , respectively for $10 \%$ heterogeneity. The second moment, which is a measure of peak broadening, increased about one order of magnitude for $10 \%$ heterogeneity. The adsorption equilibrium constant is less influential on the elution peak than the heterogeneity.

The moments are also shown in Fig. 2 as $\mu_{1} \% \theta$, $\left(P e / 2 \theta^{2}\right) \mu_{2}$, and $\left(P e^{2} / 12 \theta^{3}\right) \mu_{3}$ versus $\alpha \Delta$ in terms of $P e$ for $K=100$. The influence of heterogeneity is greater for larger values of $P e$ where axial dispersion is less important, because the relative importance of heterogeneity increases with increase in $P e$. Figure 3 shows plots of $\mu_{1}{ }^{\prime} / \theta, \mu_{2} / \theta^{2}$, and $\mu_{3} / \theta^{3}$ versus $P e$. The effect on the peak broadening is more obvious in these plots than in Fig. 2. The ordinates in Fig. 2 include $P e$ to show the variation from the homogeneous membrane, while the ordinates in Fig. 3 directly relate to the peak broadening or skewness. The second and third moments decrease significantly as $P e$ increases. As the influence of axial dispersion decreases with increase in $P e(P e>10)$, the 


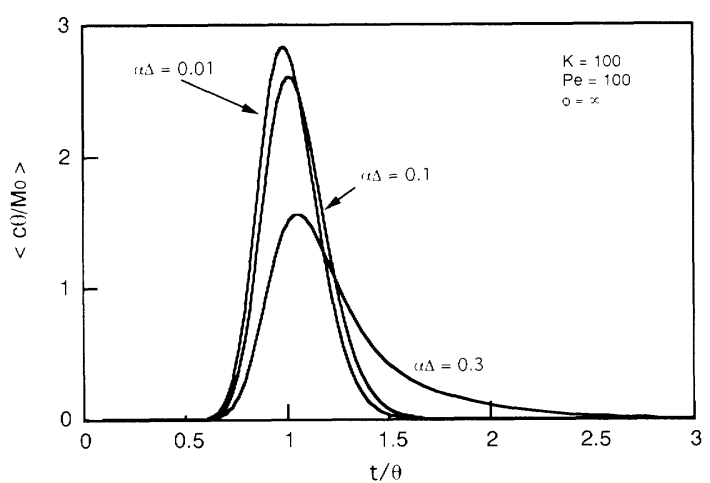

Fig. 5 Effect of heterogeneity $\alpha \Delta$ on the impulse response curve

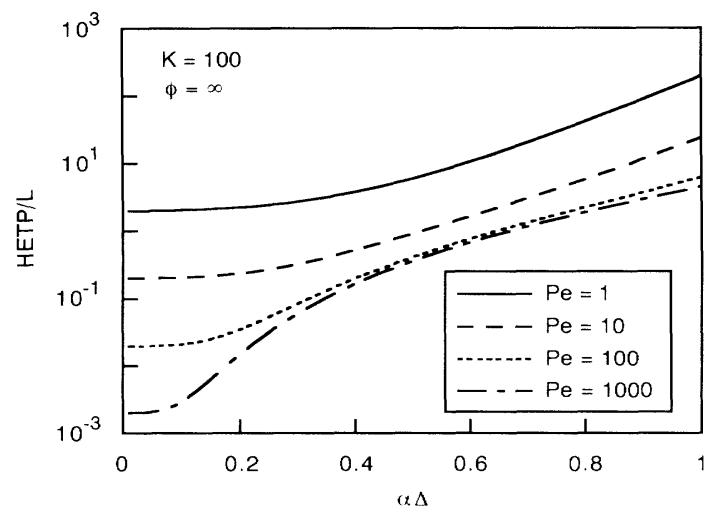

Fig. 6 Effect of heterogeneity on the HETP at different values of $P e$

influence of heterogeneity becomes dominant.

Figure 4 shows the moments for the non-equilibrium case where the kinetic parameter $k_{a}$, that is $\phi$, is accounted for. The moments are calculated for $K=100$ and $P e=100$. When $\phi$ is small, the effect of the adsorption rate controls the peak shape. When $\phi$ is larger than 10 , heterogeneity becomes influential.

The effects of heterogeneity on the chromatographic peak are visualized in Fig. 5 at three values of heterogeneity $\alpha \Delta$ for the equilibrium case. These curves were calculated by Eq. (29). As indicated by moment analyses the retention time, peak width, and skewness are considerably affected by heterogeneity.

The performance of an adsorbent column is often characterized by the concept of the height equivalent to a theoretical plate (HETP) for evaluating chromatographic processes. The HETP can be related to the moments by

$$
H E T P=L \mu_{2} / \mu_{1}{ }^{2}
$$

The HETP is calculated by the moments for various $P e$ and $\phi$. The effects of heterogeneity and axial dispersion on the HETP/L are shown in Fig. 6 for the equilibrium case. The effects of heterogeneity and the adsorption rate are shown in Fig. 7. Without heterogeneity the HETP increases as $P e$ or $\phi$ decrease. The dependence on $P e$ or $\phi$ decreases as the heterogeneity increases.

The above results revealed the substantial effect of

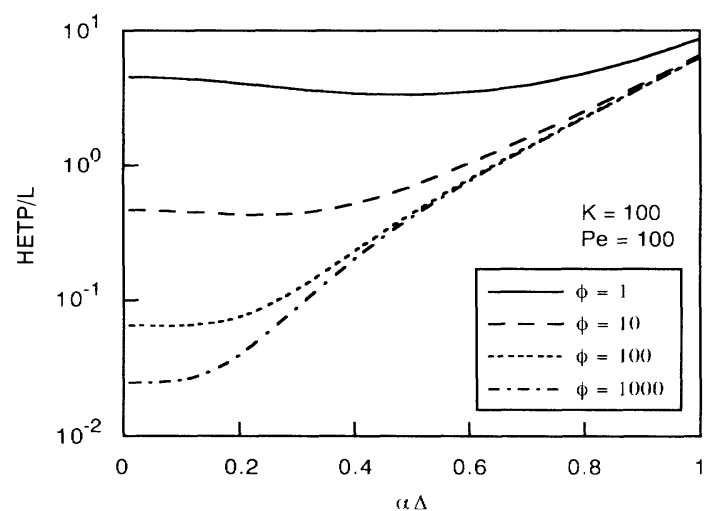

Fig. 7 Effect of heterogeneity on the HETP at different values of $\phi$

heterogeneity on frontal or peak broadening. When the axial dispersion and adsorption rates are less influential, the influence of heterogeneity is not negligible. These behaviors might be misinterpreted as peak broadening due to dispersion, mass transfer, or other rate processes. The above discussion, developed by moment analysis of a linear isotherm system, confirmed the results of Suen and Etzel ${ }^{11)}$ where the effect of heterogeneity on the breakthrough curves were shown for a nonlinear isotherm system with negligible axial dispersion. Although the moment analysis is restricted to a linear isotherm system, it has the advantage that the quantitative discussion gives us insight into the adsorption behavior. The behavior of a nonlinear isotherm system may be qualitatively estimated from the results for a linear isotherm.

Heterogeneity in membrane thickness and pore size are discussed in Appendix.

\section{Conclusion}

The heterogeneity of the membrane adsorber was examined by means of moment analysis. The moment equations were derived for heterogeneity in porosity, membrane thickness, or pore size. The calculated results were shown in the case of porosity heterogeneity. The heterogeneity produced unfavorable peak broadening and skewness. These phenomena might result in the reduction of the loading capacity of the adsorber and its resolution.

\section{Appendix}

1) Heterogeneity in thickness We consider that heterogeneity exists in membrane thickness with uniform porosity. Since the interstitial velocity is influenced by the thickness at constant pressure drop and porosity, the velocity is a function of the thickness. From Eq. (11) the interstitial velocity is inversely proportional to the thickness.

$$
v_{i}=v_{0} z_{0} / z_{i}
$$

where $v_{0}$ is the velocity at membrane thickness $z_{0}$.

The moments are derived as in the case of porosity distribution.

$$
m_{1 i}\left(z_{i}\right)=m_{0 i} \theta Z_{i}^{2}
$$




$$
\begin{aligned}
& m_{2 i}\left(z_{i}\right)=m_{0 i} \theta^{2} Z_{i}^{2}\left(\frac{2 K^{2} E}{\phi A^{2}}+\left(\frac{2}{P e}+1\right) Z_{i}^{2}\right) \\
& m_{3 i}\left(z_{i}\right)=m_{0 i} \theta^{3} Z_{i}^{2}\left(\frac{6 K^{3} E}{\phi^{2} A^{3}}+\frac{12 K^{2} E}{\phi \mathrm{A}^{2}}\left(\frac{2}{P e}+1\right) Z_{i}^{2}+\right. \\
& \left.\left(\frac{12}{P e^{2}}+\frac{6}{P e}+1\right) Z_{i}^{4}\right)
\end{aligned}
$$

where $E=(1-\varepsilon) / \varepsilon, A=1+E K, P e=z_{0} v_{0} / D_{a}, \phi=k_{a} z_{0} / v_{0}$, and $Z_{i}$ $=z_{i} / z_{0}$.

The normalized moments are obtained from these relations.

$$
\begin{gathered}
\mu_{1}^{\prime}(z)=\theta\left\langle z^{2}\right\rangle \\
\mu_{2}(z)=\theta^{2} \frac{2}{P e}\left\{\left\langle Z^{4}\right\rangle+\frac{P e K^{2} E}{\phi A^{2}}\left\langle Z^{2}\right\rangle+\frac{P e}{2}\left[\left\langle Z^{4}\right\rangle-\left\langle Z^{2}\right\rangle{ }^{2}\right]\right\} \\
\mu_{3}(z)=\theta^{3} \frac{12}{P e^{2}}\left\{\left\langle Z^{6}\right\rangle+\frac{P e^{2} K^{2} E}{2 \phi A^{2}}\left(\frac{K}{\phi}\left\langle Z^{2}\right\rangle+\frac{2}{P e}\left\langle Z^{4}\right\rangle+\right.\right. \\
\left.\left[\left\langle Z^{4}\right\rangle-\left\langle Z^{2}\right\rangle^{2}\right]\right)+\frac{P e}{2}\left[\left\langle Z^{6}\right\rangle-\left\langle Z^{4}\right\rangle\left\langle Z^{2}\right\rangle\right]+ \\
\left.\frac{P e^{2}}{12}\left[\left\langle Z^{6}\right\rangle-3\left\langle Z^{4}\right\rangle\left\langle Z^{2}\right\rangle+2\left\langle Z^{2}\right\rangle\right]\right\}
\end{gathered}
$$

A similar discussion can be developed as in the case of porosity heterogeneity.

2) Parallel pore model with pore size distribution When the pores in the membrane are represented by straight parallel pores rather than uniform porous material, the adsorbed-phase concentration would be better represented on a surface area basis. The continuity equation, Eq. (1) is replaced by

$$
\varepsilon \frac{\partial c}{\partial t}+\varepsilon v \frac{\partial c}{\partial z}=\varepsilon D_{a} \frac{\partial^{2} c}{\partial z^{2}}-\frac{2 \varepsilon}{r} \frac{\partial c_{m}}{\partial t}
$$

where $c_{m}$ has a unit of $\left[\mathrm{mol} / \mathrm{m}^{2}\right.$-surface area].

Consider the pore size $r$ has a distribution whereas the porosity is uniform. Since the flow in pores can be assumed to be in the laminar region, the interstitial velocity within the pore is given by

$$
v_{i}=\left(r_{i} / r_{0}\right)^{2} v_{0}
$$

where $v_{0}$ is the velocity in the pore of $r_{0}$.

The derivation of the moment equations developed above is modified to give the moment expression for pore size distribution. The moment equations Eqs. (14)-(17), (22), (25) and (26) are applicable in this case too when parameters are defined by $E_{i}=2 / r_{i}, E_{0}=2 / r_{0}$, and $B_{i}\left(r_{i} / r_{0}\right)^{2}$. The other factors which may cause heterogeneity can be evaluated in a similar manner.

\section{Acknowledgment}

The authors are grateful to Professor Benjamin J. McCoy (University of California, Davis, USA) for helpful discussion.

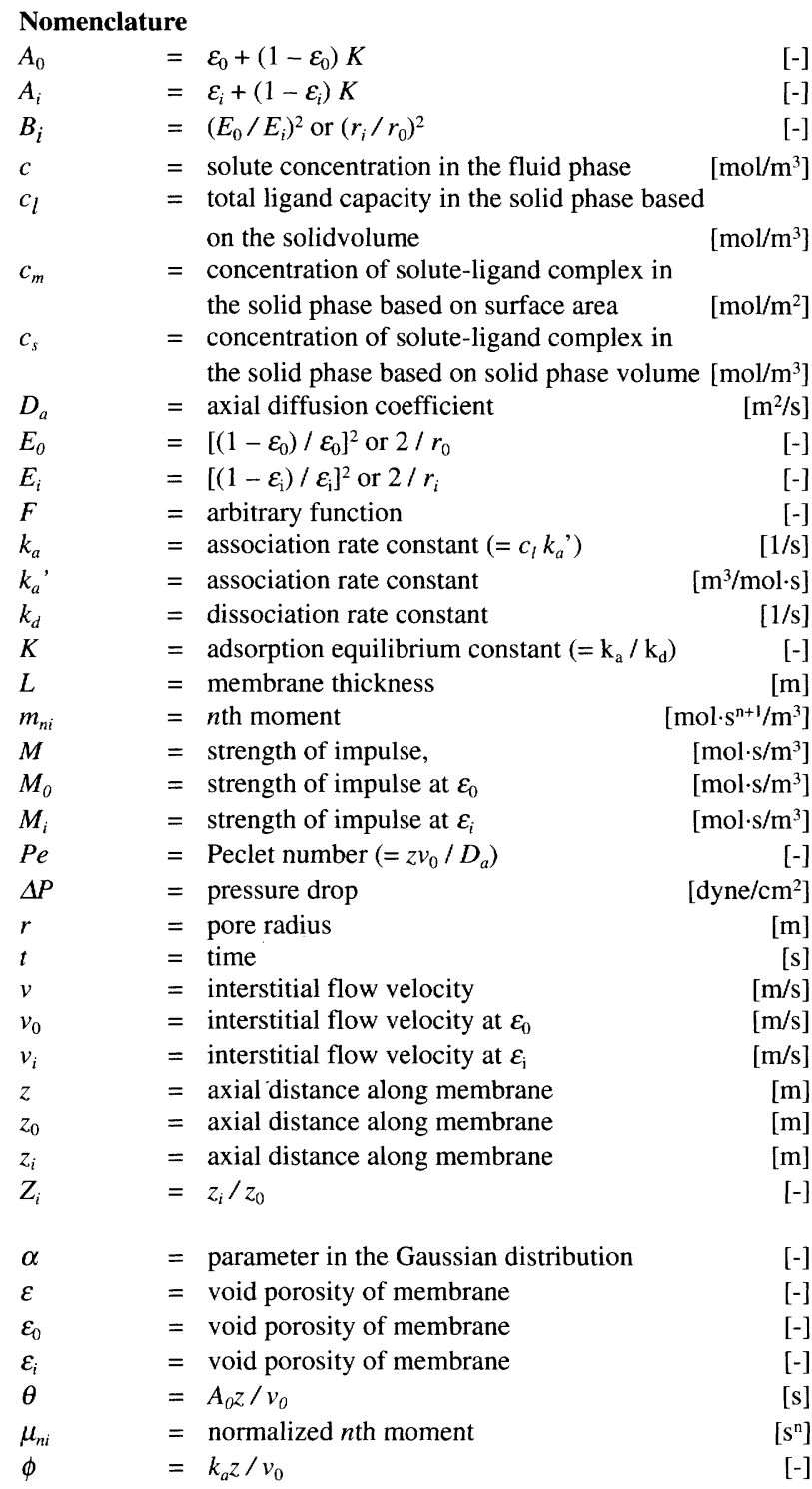

\section{Literature Cited}

1) Arnold, F. H., S. A. Schofield and H. W. Blanch: J. Chromatogr. 355, 1-12 (1986).

2) Binous, H. and B. J. McCoy: Chem. Eng. Edu., 26, 12-17 (1992).

3) Bird, R. B., W. E. Stewart and E. N. Lightfoot: "Transport Phenomena", Wiley, New York (1960).

4) Brandt, S., R. A. Goffe, S. B. Kessler, J. L. O'Connor and S. E. Zale: Bio/Technology, 6, 779-782 (1988).

5) Briefs, K. G. and M. R. Kula: Chem. Eng. Sci., 47, 141-149 (1992).

6) Klein, E.: "Affinity Membranes", John Wiley \& Sons (1991).

7) Konishi, S., K. Saito, S. Furusaki and T. Sugo: Ind. Eng. Chem. Res., 31, 2722-2727 (1992).

8) Levenspiel, O. and J. M. Smith: Chem. Eng. Sci., 6, 227 (1957).

9) McCoy, B. J.: Chem. Eng. Sci., 44, 993-996 (1989).

10) Rodrigues, A. E., J. C. Lopes, Z. P. Lu, J. M. Loureiro and M. M. Dias: J. Chromatogr., 590, 93-100 (1992).

11) Suen, S. Y. and M. R. Etzel: Chem. Eng. Sci., 47, 1355-1364 (1992). 